\title{
Team YowAI-2001 Description
}

\author{
Koji Nakayama and Ikuo Takeuchi \\ Department of Computer Science \\ The University of Electro-Communications \\ Chofu, Tokyo 182-8585, Japan \\ robocup@takopen.cs.uec.ac.jp
}

\section{Introduction}

The team YowAI-2001 inherits the low-level individual skill and the world modeling technique of YowAI-2000 developed by Takashi Suzuki and improved by Sinnosuke Asahara. Its objective is "cooperation by short shouts" instead of cooperation by communicating large amount of non human-like messages among agents.

Our former team YowAI-2000 puts emphasis only on the quality of low-level individual skill and world modeling, but it does not involve explicit cooperation among agents. For example, YowAI-2000 uses neither say command nor hear command at all. It uses a fixed positioning strategy. However, YowAI-2000 won the victory in RoboCup Japan Open 2000, and obtained the 6th place in RoboCup 2000 held in Melbourne.

From these result, it can be said that each agent's individual low-level skill and world-modeling accuracy have prior importance to cooperation. However, it is also true that it is difficult to achieve further improvement in the performance of a multi-agent system only by the improvement in agent's individual low-level skill and world-modeling accuracy. Therefore, YowAI-2001 tries to adopt action decisions based upon tactics and strategy of skillful human soccer players. Here, the most important criterion of YowAI-2000 is that it uses only communication by short shouts as seen (or heard) in human soccer play.

The main purpose of YowAI-2001 as a research topic in distributed AI is that how far man-like communication can realize cooperation, without using detailed numerical or symbolic information such as absolute coordinate and elaborate plan sequence. We believe that powerful cooperation in RoboCup should be realized only by minimum utterance.

\section{Team Play by Shouts}

The communication between agents should be transmitted by the soccer server. If an agent who wants to communicate publishes say command to the server, the server transmits hear command to agents within 50 meter distance from the utterer. Detailed coordinates and numerical information can be told because of the comparatively loose current regulation that a say command is a sequence of less than 512 (almost any kind of) ASCII characters. 
However, say commands used in YowAI-2001 are much simpler as shown below. We are now extending the vocabulary based upon common soccer tacitics and strategy.

\section{(say SwordFish shotteru 4)}

This message means that an opponent player is pressing behind the player No. 4. Here, "SwordFish" is the nickname of YowAI-2001 in the Takeuchi Laboratory of the University of Electro-Communications.

In actual soccer, players use minimun communication methods such as a shout and eye contact. This shows that the efficiency of communication between players is important in soccer. For example, the shout "AGERO" is used in the meaning "push up a defense line." So, players do not say concretely, "push up a defense line." Becase the length of the word which man can understand immediately is one or two. If players say concretely, the player who is said cannot understand immediately and cannot take action. Note the important thing that players can communicate tactically enough without adding any concrete word.

The essence of shout is that gives a tactical self/partner's situation immediately and gives a hint which is selection of judgment or action for partner. Selection of judgment or action is not forced. The shout must be short, the given tactical situation have to be highly abstracted concept with an understanding common to teammates.

Since the shout is highly abstracted, both of player who uses the shout have to refer to own world model or the strategy plan which the whole team should have in common. Therefore, in order to perform cooperation only by the shout, it is necessary to make each agent's intelligence into high level from the case where extensive information communication is possible. In addition, also have to consider quality of a shout. For example, as for the shout "pass" any teams are used abundantly. However, the tactical judgment of the player who said, "pass" may be worng. As it becomes a strong team, the reliability of a thing which "pass" means becomes higher.

\section{1 shotteru}

"SHOTTERU" is shouted when one or more opponent players approach behind our player who is going to receive the ball. The player who hears this voice uses its ball keeping skill, and prevents taking a ball if he is in the opponent's field and showing his back to the opponent's goal. Other players occasionally move to supporting position and say "HATAKE" which will be described immediately below. This simple message may raise various action decisions depending on the players condition.

\section{2 hatake}

"HATAKE" is shouted from a player who is in the position who can receive a short pass from a "SHOTTERU" player rather safely. Then, the "SHOTTERU" 
player who hears this voice will pass to the direction of the voice if he decides it is reasonable.

\section{3 gotzan}

"GOTZAN" is shouted by a player who is in a better position for shooting than who is now controlling the ball.

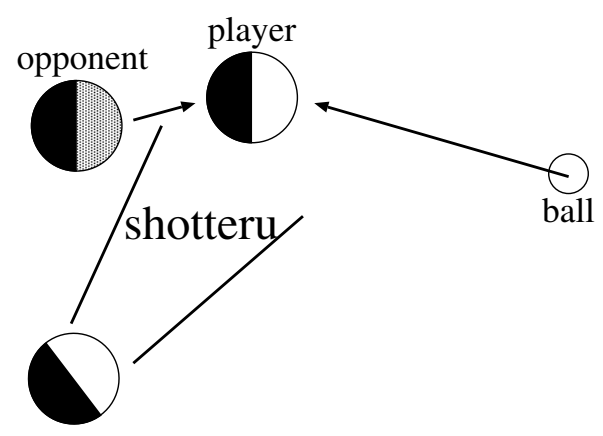

Fig. 1. shotteru

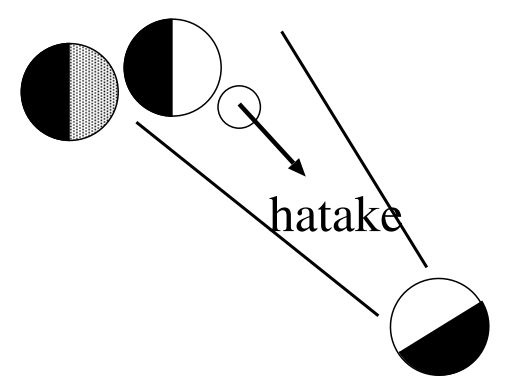

Fig. 2. hatake
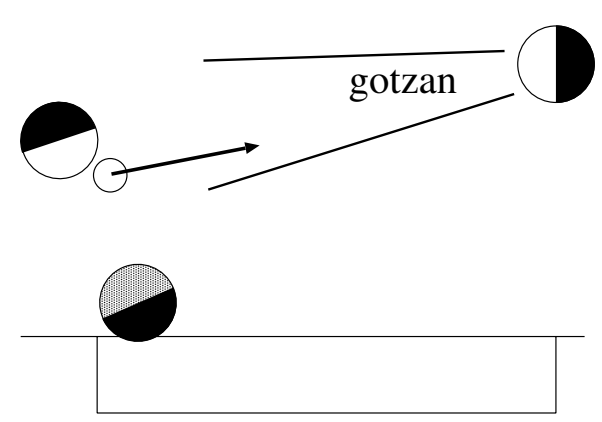

Fig. 3. gotzan 


\section{Future Work}

We have to extend the vocabulary of shouts. The vocabulary shown above is obviously too small to make agent's communication more effective and "organic". We shall limit the usage of numerical value to minimum, that is, only small positive integers.

It should be noted here that improvement of individual tactical and strategic ability is important to make such short shouts work well. This improvement will be our main future research. 